\title{
Does Sri Lanka's Drug and Laboratory Supply related Regulatory Mechanism address the current concerns at tertiary level hospital care?
}

Gunasekera V.

\begin{abstract}
Although medicinal products and drugs may have similar characteristics as any other commodity, consumers would need specific knowledge to make decisions with regard to their usage, benefits, harms and potential risks. Poor regulatory capacity could lead to monopolies, breach of market principles, market failure, trade ethics and large scale corruption. If unregulated, money spent for procuring and providing poor quality medicinal products, which are unsafe and ineffective may go down the drain.
\end{abstract}

However, institutional level Health Technology Assessment (HTA) has been given little importance in the current day practice specifically in Tertiary level hospitals. Due to lack of capacity at institutional level for evidence based decision making, tertiary level institutions may not be able to make right choices to produce desired clinical outcomes.

Therefore, regulatory needs of large institutions such as tertiary level care institutions and specialized hospitals need to be addressed with special emphasis on their uniqueness and specific needs. Country's drug and medicines regulatory mechanism is paramount in fulfilling fundamental public health objectives.

\section{Introduction}

Unlike for any other commodity medicinal products and drugs, consumers would need specific knowledge to make decisions with regard to their usage, benefits, harms and potential risks. Therefore, professional advice is needed to make this consumer decision from a prescriber or the dispenser in order to make the right choice.

\section{Why regulation is important}

There may be many consequences resulting from using poor quality and harmful drugs such as treatment failure and worsening disease conditions, drug resistance especially in the case of antibiotics and even death. Use of poor quality drugs may also demonstrate a bad impression on the health care delivery system and create frustration among professionals, practitioners, and all other industry stake holders. Furthermore, poor regulatory capacity could lead to monopolies, breach of market principles, market failure, trade ethics and large scale corruption. Resources used for procuring and providing poor quality medicinal products, which are unsafe and ineffective may go down the drain.
Therefore, it is the government's responsibility to protect its citizens/patients in this specific field where they would not have adequate knowledge, skills to make right consumption decision. In order to safeguard the public from such ruinous situations, government need to establish a strong mechanism for drug and medicinal product regulation institutionalizing a body of strong National Medicinal and Drug Regulatory Authority (NMRA). In broader perspective, the body should be able to regulate, promote and protect all public health aspects of drugs and medicinal products in the country. Hence the mechanism will need strong technical capacity in terms of knowledge, skills and capacity to make scientific evidence base decisions on these products within a well-structured legal frame.

\section{Minimum regulatory functions for a National Regulatory Authority (NRA)}

As an absolute minimum NRAs should:

- ensure that all medicines manufacturing, importation, exportation, wholesale and distribution establishments are licensed.

Activities and premises must comply with Good Manufacturing Practices (GMP) and Good Distribution Practice requirements.

- before medicines are marketed, assess their safety, efficacy and quality.

- monitor the quality and safety of medicines on the market to prevent harmful, substandard and counterfeit medicines from reaching the public.

- regularly inspect and control the informal market, including e-commerce, to prevent illegal trade of medicines.

- monitor advertising and promotion of medicines, and provide independent information on their rational use to the public and professionals.

- participate in sub-regional and regional regulatory networks and international meetings of drug regulatory authorities to discuss issues of mutual interest and concern, facilitate timely exchange of information and promote collaboration.

- monitor and evaluate performance to assess if perceived regulatory objectives have been met, to identify weaknesses and take corrective action.

Source: WHO Policy Perspectives on Medicines no 7, 2003. 


\section{Resources for Drug Regulation.}

Tertiary level care hospitals are institutions that have special needs which should be addressed. Therefore, tertiary level hospitals needs to be equipped with mini Health Technology Assessment (HTA) hubs that would liaise with country's main regulatory body in the case of Sri Lanka it's the NMRA, where this interaction would address some information gaps and capacity issues.

Drugs and medicinal regulatory affairs need a strong capacity in terms of expertise, infrastructure, laboratory capabilities and strong information technology back bone. Sri Lanka is currently facing severe shortages of qualified experts to evaluate drugs and medical devices. However, it is the situation in many low resource settings and therefore way out would be to develop resilient networks with other agencies such as US Food \& Drug Administration (US FDA), UK National Institute for Clinical Care Excellence (UK NICE), and European Medicines Agency (European Commission, Regulation, 2004). Constant exchange of information and developing higher level analytical skills to utilize existing knowledge would save our time and more importantly prevent duplication.

Current capacity of the drug testing laboratory and inefficiencies due to various routine processes have to be rectified urgently as laboratory capabilities and capacity is paramount in drug regulatory activities. Sri Lanka is currently facing serious drawbacks in this area leading to delays in testing new chemical entities or new products entering the market, inefficient testing of failed and withdrawn products and ineffective post marketing surveillance.

NMRA needs a robust information support which should be founded on a well-organized information management system. This may streamline all activities related to NMRA and the system will be more Industry friendly, transparent and efficient.

\section{Scientific, evidence based decision making is needed at tertiary level care.}

Health Technology Assessment (HTA) is a key strategy to bring in evidence to make informed decisions. However, institutional level HTA assessment has been given little importance in the current day practice specifically in tertiary level care hospitals. Strong regulatory networks within and outside the region would be important to share knowledge. Developing HTA capacity in the country will certainly be important not for carrying out in depth analysis and appraisals each time, but to interpret and make information adaptable to local situations.
More investments should direct towards such capacity enhancement and more attention should be paid in developing capacity such as Good Manufacturing Practices (GMP) and Good Clinical Practices (GCP), how to assess generic medicines, equivalence, safety monitoring and pharmacovigilance, quality assurance and quality control.

\section{Should regulation only cover drugs and devises?}

Although drugs and medical devices have gained more attention and falls under a strict regulatory framework, much attention is needed to regulate blood and blood products and cosmetics in Sri Lanka. Very little has been done on these aforesaid areas and as a result Sri Lanka has not been able to appropriately regulate blood related products which is considered as a pharmaceutical product in many other countries and cosmetics and related products which can cause serious harmful effects to citizens.

\section{Way forward}

Building capacity and enhancing professional expertise would be a major priority area in future expansion of drug regulation in Sri Lanka. Globally there seems to be a severe dearth of human resource in this area and therefore, strong collaborations, harmonization and effective sharing of information would optimize overall regulatory performance of the country. Furthermore, institutional level appraisal of drugs and laboratory technologies would be the way forward in dealing with tertiary level care related decision making.

Use of a state-of-the-art information technology platform for all processes related to regulatory cycle will be a challenge in the current context as there will be lot of resistance to change arising within the system. (Goroshenko,1996) However, working towards establishing such a systematic process-oriented work flow and bringing in industry ethics within a sound legal frame would certainly benefit overall regulatory environment in the country.

Upgrades and improvements are essentially needed for the drug testing laboratory and taking the technology to the next level is a mandatory requirement to enhance laboratory capacity that will cater to the current demand and regulatory needs.

Strong legal framework should be established in order to promote best practices in the industry. Hence, the current legal framework governing medical products for human use, should include all related products such as blood and blood products (Klein. R, 1995). 
Another area of challenge includes new technologies and emerging treatment modalities. Fast developing technologies of Pharmacogenetics, biological medicinal products and nanotechnologies are few examples for such development. A "complete rethink" may be necessary in terms of regulation to deal with these new trends.

It is the ultimate responsibility of the government to ensure quality, safety and efficacy of all medicinal products and provide cost effective options which would be affordable to both public and to the health care delivery system. Regulatory needs of large institutions such as tertiary level care institutions and specialized hospitals need to address with special emphasis on their uniqueness and specific needs. Country's drug and medicines regulatory mechanism is fundamental in achieving this core public health obligation.

\section{References}

1. https://www.who.int/medicines/publications/ policyperspectives/en/

2. European Commission. Regulation (EC) No 726/2004 of the European Parliament and of the Council of 31 March 2004 laying down Community procedures for the authorisation and supervision of medicinal products for human and veterinary use and establishing a European Medicines Agency.

3. Goroshenko, B., Volovei, V. and Mochniaga, A. (1996) Health Care Systems in Transition: Republic of Moldova. Copenhagen: European Observatory on Health Care Systems.

4. Klein, R. (1995) Priorities and rationing: pragmatism or principles?, British Medical Journal, 311: 761-2. 